\title{
Observation of Cosmic Rays with Nuclear Emulsions inside Egyptian Pyramids
}

\author{
Kunihiro Morishima ${ }^{1}$ \\ Institute for Advanced Research, Nagoya University \\ F-lab., Department of Physics, Nagoya University, Furo-cho, Chikusa, Nagoya, Aichi, Japan \\ E-mail: morishimalflab.phys.nagoya-u.ac.jp
}

\section{Mitsuaki Kuno}

Department of Physics, Nagoya University

\section{Akira Nishio}

Department of Physics, Nagoya University

\section{Yuta Manabe}

Department of Physics, Nagoya University

\section{Nobuko Kitagawa}

Institute of Materials and Systems for Sustainability, Nagoya University

\begin{abstract}
A nuclear emulsion is a three dimensional tracking detector which is able to record minimum ionizing particles with micrometric accuracy. We are developing the nuclear emulsion for observation of cosmic rays and its analysis techniques. We have been applying nuclear emulsions to cosmic ray radiography for measuring the inner structure of nuclear power plants, volcanoes and so on. Since 2015, we have been observing cosmic rays inside the pyramids, which are the Bent Pyramid at Dahshur and Khufu's Pyramid at Giza, in Egypt through ScanPyramids. In this project, we produced nuclear emulsions and installed them into the pyramids to measure cosmic ray muons. We observed over ten million muons passed through the pyramids and reconstructed the angular distribution of detected muons. Through the analysis, the validity of cosmic ray radiography for investigation of a pyramid was confirmed and the new space inside the Khufu's Pyramid was discovered. In this paper, nuclear emulsions for the observation and latest results are described.
\end{abstract}

\section{${ }^{1}$ Speaker}




\section{Nuclear Emulsion}

Cosmic ray radiography is a visualization technique of internal structure of large-scale objects by observing cosmic ray muons from inside or outside of target object. One of the first experiments was done by L. W. Alvarez to search for hidden chambers in the Khafre's Pyramid at Giza [1]. The principle of the method is the measurement of attenuation of cosmic ray muons in matter. After L. W. Alvarez's experiment, measurements of volcano [2] and nuclear reactor [3, 4] have been successfully conducted recently.

In order to observe cosmic rays for that purpose, the detector is requested for measurement of three dimensional direction of trajectory of muon. In addition, in order to achieve higher imaging resolution and better signal noise ratio, larger statistics of detected muons is necessary. But the flux of cosmic ray muons on the ground is almost constant. Thus, a large area detector is required to collect more muons. From these kinds of reasons, requirements for the detector are both high directional accuracy and large area for statistics.

A nuclear emulsion is suitable for these requirements from the following properties. A nuclear emulsion is able to detect a trajectory of a minimum ionizing particle with submicrometric accuracy. Thanks to this high spatial resolution, a nuclear emulsion is able to measure angle of trajectory of muons with angular accuracy of an order of mrad. In addition, the thickness is less than $1 \mathrm{~mm}$. Furthermore, a nuclear emulsion doesn't need electric power supply and easy to enlarge the detector area. These properties are ideal for usage of cosmic ray radiography.

Until now, we used nuclear emulsions for cosmic ray radiography of volcanoes, nuclear reactors and so on $[2,3]$. We are conducting cosmic ray radiography of the pyramids at Egypt through ScanPyramids, which is an international project of investigation of pyramids, since 2015 [5]. In this paper, we present current status of observation of cosmic ray inside the pyramids for cosmic ray radiography.

A structure of a nuclear emulsion is shown in Fig. 1. There are a lot of silver bromide crystals, which are sensitive to minimum ionizing particles, dispersed in a gelatin layer. A trajectory of muon recorded in two sensitive layers, which are coating on both sides of a supporting plastic base, is called track. Tracks are read out by the automated scanning system "Track Selector" and they are saved as digital information into computer storage devices $[6,7]$.

We introduced the emulsion gel production machine in our laboratory in 2010 for selfproduction and we are studying improvement of performance for purposes. In addition, we constructed the manual mass production system. In this way, it is possible to produce fully nuclear emulsion in our laboratory at Nagoya University [8]. By using these systems, we tuned the volume occupancy ratio of silver bromide crystals, which becomes $35 \%$, in a gelatin layer for improvement of long term stability for cosmic ray radiography. Its sensitivity, which is evaluated by a unit called grain density, is about 35 silver particles per $100 \mu \mathrm{m}$ for minimum ionizing particles and that is enough for detection of muons in an emulsion layer of the thickness of $60 \mu \mathrm{m}$.

\section{ScanPramids}

ScanPyramids is an international scientific research project, which was organized mainly by Egypt's Ministry of Antiquities and Heritage Innovation Preservation Institute (HIP) [5]. This project has started in October 2015. We are conducting cosmic ray radiography with nuclear emulsion for an investigation of inner structures of pyramids through participation in the project. 
We measured cosmic rays in the chamber inside the Bent Pyramid at Dahshur and the Khufu's Pyramid at Giza.

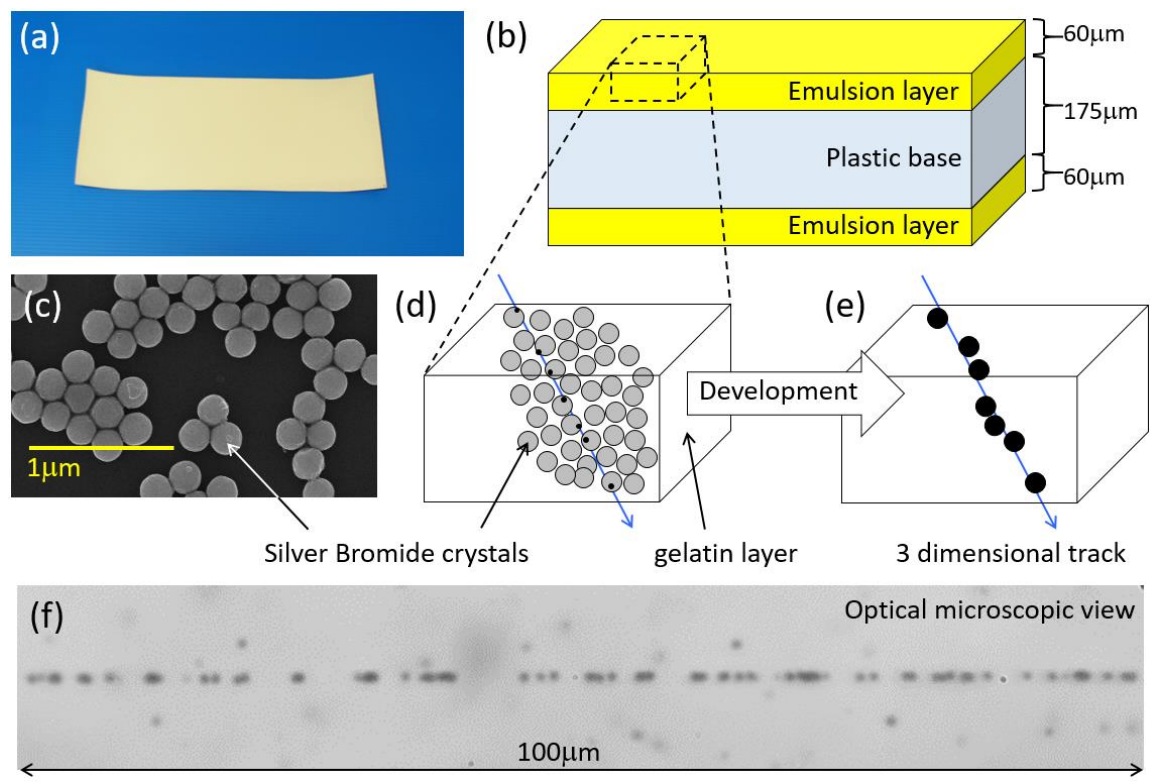

Figure 1: (a) An image of the nuclear emulsion; (b) An illustration of the structure of the nuclear emulsion; (c) An electron microscope image of silver bromide crystals of the nuclear emulsion produced at Nagoya University. The diameter of crystals is approximately $200 \mathrm{~nm}$; (d) and (e) The principle of detection of a charged particle passes through a nuclear emulsion. Black small dots on silver bromide crystals in (d) show signal which was passed by particles; (f) An optical microscope image of a track, which consists of silver particles.

\section{Experimental overview}

A pyramid is built of limestone. However, the detail inner structure of a pyramid isn't still well understood and limestone density depends on the production region. Thus, the density length of a pyramid is estimated with large uncertainty. If the average density is $2.2 \mathrm{~g} / \mathrm{cm}^{3}$ inside a pyramid, penetration rate is estimated to be about $1 \%$ after passing through limestone of the thickness of $100 \mathrm{~m}$. From such estimation, we planned the measurement period and the detector area for each installation position.

We produced nuclear emulsions of more than $70 \mathrm{~m}^{2}$ for ScanPyramids project since 2015 . We transported them from Nagoya to Cairo by an airplane without exposing X-ray and with keeping low temperature. Until now we installed them in the Bent Pyramid and in the Khufu's Pyramid. After observation period, we collected them from the pyramids and did photographic development at Great Egyptian Museum (GEM) at Giza. After that, we transported nuclear emulsions back to Nagoya University for scanning by the Track Selector.

\section{Simulation}

A simulation was done by the following step. The first step is modeling of three dimensional structures, which was done by Cairo University and HIP. The second step is calculation of the density length $\left(\mathrm{g} / \mathrm{cm}^{2}\right)$ in each direction from the detector position. The third step is calculation of the number of muons, which is able to pass through the density length in each direction, by using the model of energy spectrum of cosmic ray muons. In this analysis, we used the equation 
given by S. Miyake as a cosmic ray flux model [9]. Relation between the thickness of rock which muon can penetrate and its energy is shown in Fig. 2.

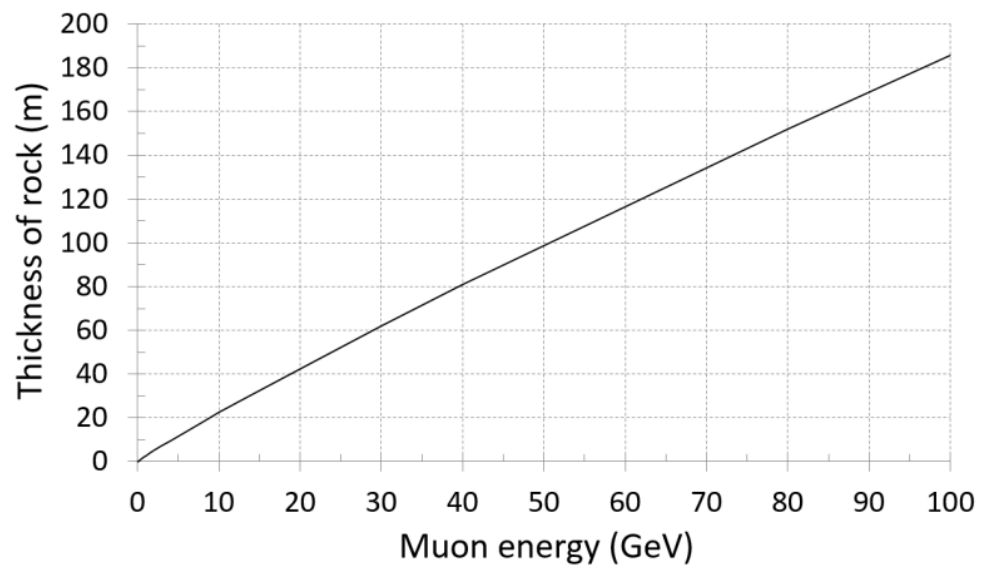

Figure 2: A relation between muon energy and the thickness of rock which density is $2.2 \mathrm{~g} / \mathrm{cm}^{3}$. The graph was made by using the table described in Ref. [10].

\section{Observation of cosmic rays inside the Bent Pyramid}

The Bent Pyramid is located at Dahshur (Fig. 3 (a)). The cross section of the Bent Pyramid is shown in Fig. 3 (b). Nuclear emulsions were installed inside the lower chamber for validation of imaging of the known upper chamber as shown in Fig. 3 (d). Fig. 3 (c) shows the detector, which is consist of 40 nuclear emulsion detectors, installed in the chamber. The area of the detector is $3 \mathrm{~m}^{2}$.

The Bent Pyramid has two entrances and two independent chambers connecting to each entrance. Nuclear emulsions were installed in the lower chamber and recorded cosmic ray muons in 40 days from December 2015 to January 2016. Through scanning by the latest scanning system called Hyper Track Selector, about $10^{7}$ tracks were read out and saved into computer storage devices. Raw data includes not only muon tracks but also other particle tracks as noise. Raw data was processed through noise reduction procedure for selection of muons.

The angular distribution of muon tracks was binned and converted to the flux for comparison with the simulation result. And then, the normalization process were done for minimizing the differences of the absolute flux due to fading effect of a nuclear emulsion. Through the process, the angular distribution of detected muons was compared with the simulation result as shown in Fig. 4. Both results show that red means a large number of detected muons and blue means a small number in two-dimensional angular space, which unit of axis is $\tan \theta$. The dashed circle in each image shows the direction of the upper chamber. Through the analysis, we confirmed excess of muon flux in the direction of the upper chamber as expected by the simulation [11].

\section{Observation of cosmic rays inside the Khufu's Pyramid}

The Khufu's Pyramid is located at Giza (Fig. 5 (a)). The cross section of the Khufu's Pyramid is shown in Fig. 5 (b). Nuclear emulsions were installed in the Descending Corridor connecting from the main entrance and the Queen's Chamber since 2016 and the measurement is still continuing. The detector, which area is $0.2 \mathrm{~m}^{2}$, in Descending Corridor and the detector, which area is $5 \mathrm{~m}^{2}$, in the Queen's Chamber are shown in Fig. 5 (c), (d). The result of observation in the 
Descending Corridor in 67 days from June 2016 to August 2016 is shown in Fig. $6.3 \times 10^{6}$ tracks were analyzed in the observation. There is a large excess compared with the simulation result in the region from the center to north side, which is pointed by a white arrow. The excess of muon flux is over five sigma and this is strongly suggesting unknown space behind the north face [12].

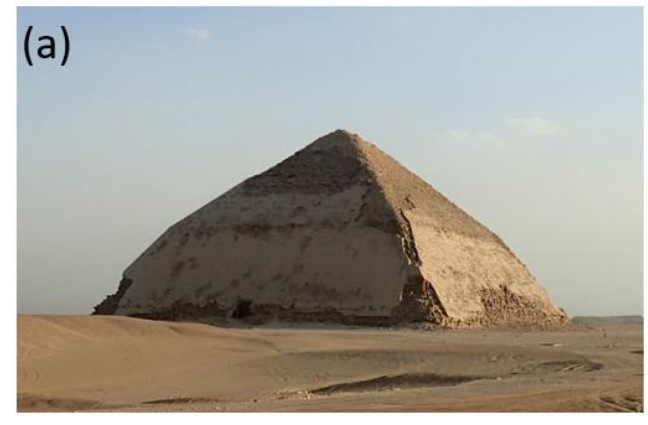

(c)

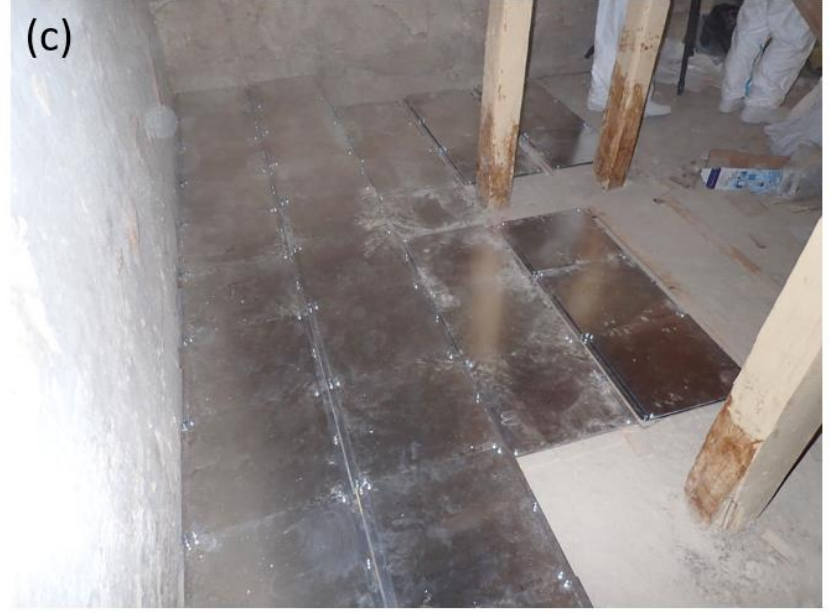

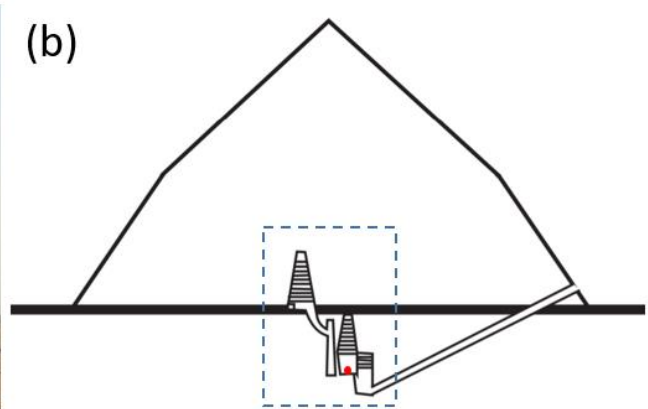

(d)

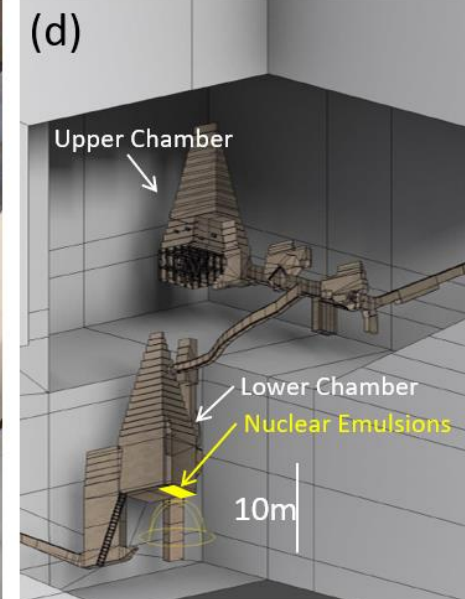

Figure 3: The observation inside the Bent Pyramid (a) An image; (b) A cross section drawing; (c) An image of nuclear emulsion detectors installed in the lower chamber; (d) An enlarged view of two chambers, which region is shown as dashed square in (b).
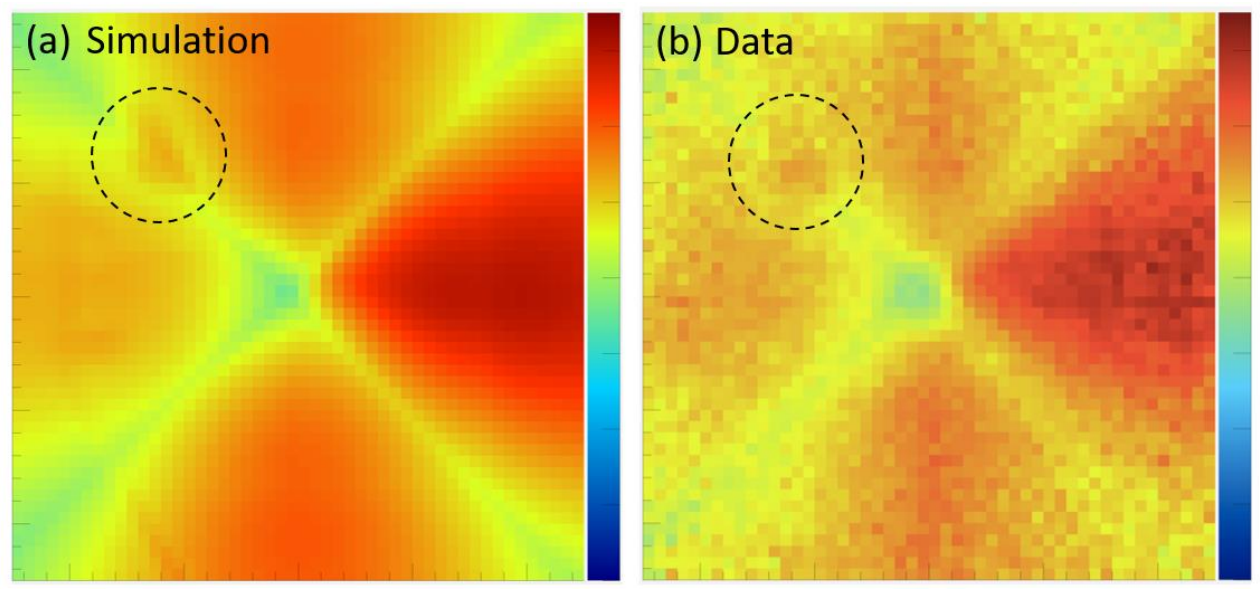

Figure 4: Angular distributions (a) The simulation result; (b) The data obtained. 

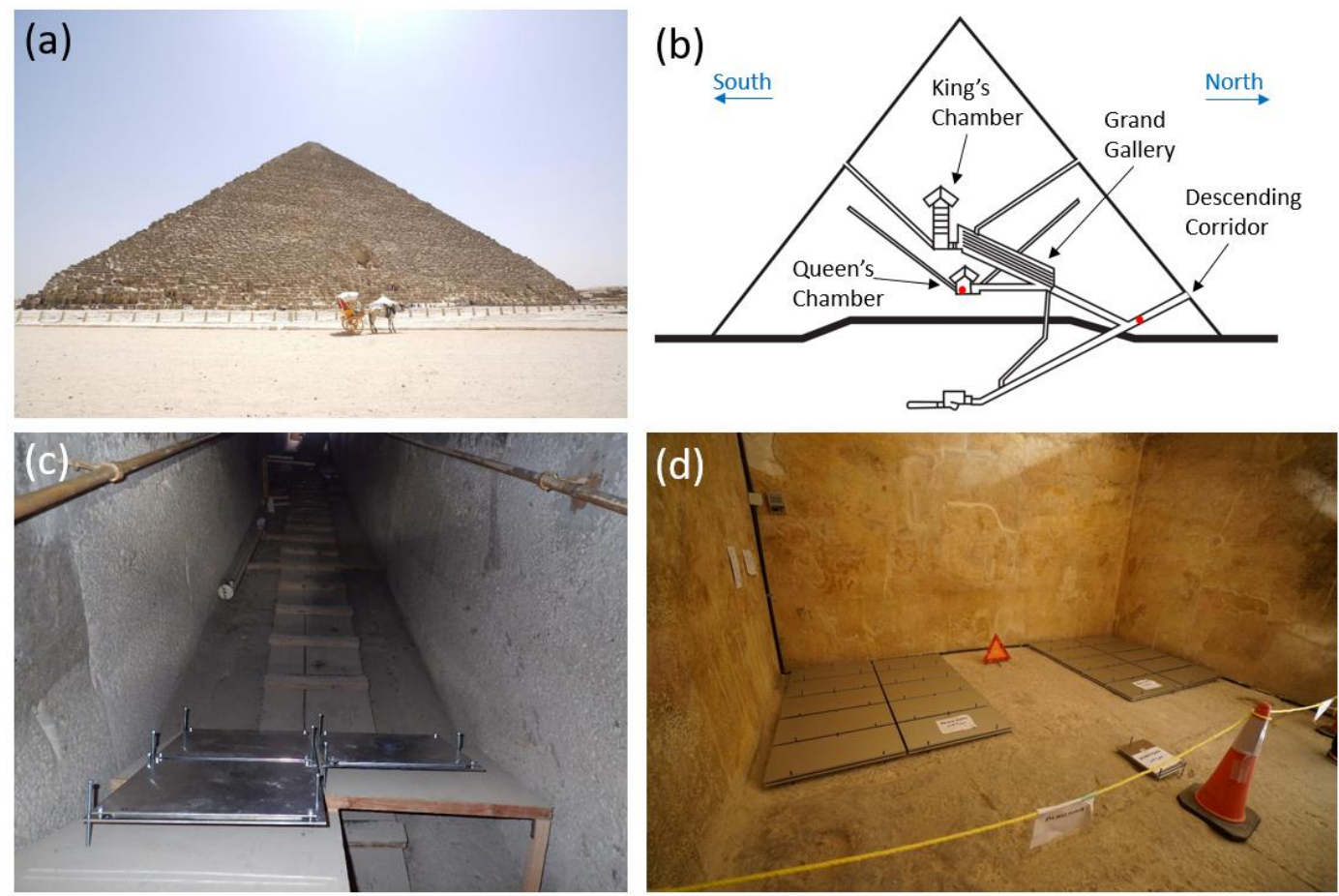

Figure 5: The observation inside the Khufu's Pyramid (a) An image; (b) A cross section drawing. Red points show installation positions; (c) An image of nuclear emulsion detectors installed in the Descending Corridor; (d) An image of nuclear emulsion detectors installed in the Queen's Chamber.
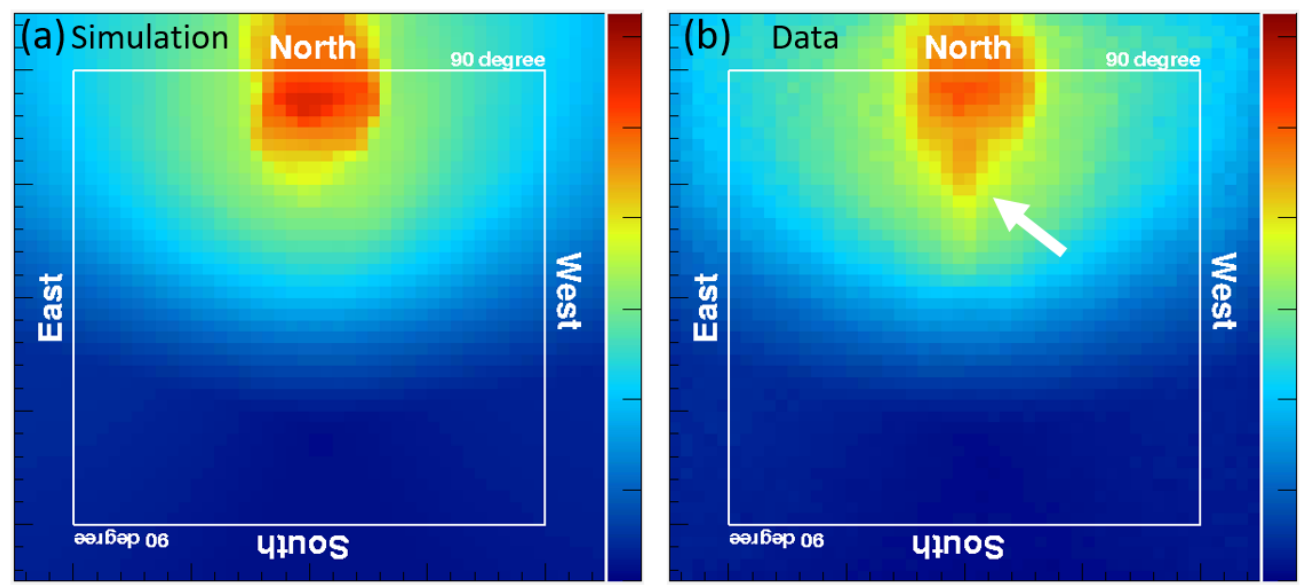

Figure 6: Angular distributions (a) The simulation result; (b) The data obtained.

\section{Conclusions}

A nuclear emulsion is an ideal detector for observation of cosmic ray muons inside a pyramid. ScanPyramids has started 2 years ago and nuclear emulsions were used in the project for searching hidden structures inside the pyramids. The validity of cosmic ray radiography for investigation of a pyramid was confirmed through the observation in the Bent Pyramid. In addition, the new space behind the north face of the Khufu's Pyramid were discovered by observing in the Descending Corridor. From these results, we are continuing observation of cosmic rays for revealing the 
structure of the new space from additional different positions in the Descending Corridor. Furthermore, we are continuing to analyze the data taken in the Queen's Chamber.

\section{Acknowledgement}

The authors would like to thank staffs of ScanPyramids. This work was supported by JSPS KAKENHI Grant Number JP15H04241.

\section{References}

[1] L. W. Alvarez et al., Search for Hidden Chambers in the Pyramids, Science 167p. 832-839 (1970).

[2] H. K. M. Tanaka et al., Imaging the conduit size of the dome with cosmic ray muons: The structure beneath Showa Shinzan Lava Dome, Geophysical Research Letters 34, L22311 (2007).

[3] K. Morishima et al., "First demonstration of cosmic ray muon radiography of reactor cores with nuclear emulsions based on an automated high-speed scanning technology", RADIATION DETECTORS AND THEIR USES Proceedings of the 26th Workshop on Radiation Detectors and Their Uses in KEK, 27-36 (2012).

[4] H. Fujii et al., Performance of a remotely located muonradiography system to identify the inner structure of a nuclear plant, Progress of Theoretical and Experimental Physics. 073C01 (2013).

[5] http://www.scanpyramids.org

[6] K. Morishima et al., Development of a new automatic nuclear emulsion scanning system, S-UTS, with continuous $3 D$ tomographic image read-out, Journal of Instrumentation 5, P04011 (2010).

[7] K. Morishima et al., Development of Automated Nuclear Emulsion Analyzing System, Radiation Measurements 50, 237-240 (2013).

[8] K. Morishima, Latest Developments in Nuclear Emulsion Technology. Physics Procedia 80, 19-24 (2015).

[9] S. Miyake., Denver, in proceedings of 13th International Cosmic Ray Conference 5, p. 3638 (1973).

[10] D. E. Groom et al., Atomic Data and Nuclear Data Tables, 78, 183 (2001).

[11] http://www.hip.institute/press/HIP_INSTITUTE_CP7_EN.pdf

[12] http://www.hip.institute/press/HIP_INSTITUTE_CP9_EN.pdf 